\title{
Determination of the interaction impedance of helix slow-wave structures
}

\author{
Richard G. Carter \\ Engineering Department, Lancaster University, Lancaster LA1 4YR, UK \\ Phone: +44 1524 593086, FAX +44 1524 594207, e.mail: r.carter@lancaster.ac.uk
}

The computer simulation of helix travelling-wave tubes requires accurate information about the phase velocity, interaction impedance and attenuation of the helix slow wave structure at each frequency. This has received considerable attention, from the pioneering work of Pierce onwards, and it is possible to compute or measure the phase velocity with sufficient accuracy. The methods available for determining the interaction impedance, however, cannot yet give good enough results and there are appreciable differences between the figures given by different methods. The purpose of this paper is to review the assumptions and approximations which are made in each case with a view to finding ways of achieving agreement between them.

There are, essentially, three ways to determine the properties of a helix slow-wave structure whose dimensions are known: mathematical analysis, computer simulation and experiment. This paper is restricted to structures with tape helices supported by dielectric rods in a cylindrical conducting shell.

Mathematical analysis: is difficult because the helical structure does not readily fit any simple co-ordinate system. The most recent publication is that by D'Agostino et al. [1]. The method assumes continuity of the tangential electric field on the surface of the helix and that the tape is perfectly conducting in the winding direction. Good convergence of the results was obtained by including at least 5 space harmonics and using at least 30 concentric dielectric regions to model the support rods. The use of an effective relative permittivity for each dielectric region effectively assumes that the tangential component of the electric field is zero. When compared with experimental results the method showed accuracies of $10 \%, 15 \%$ and $25 \%$ for three different structures. The details of the experimental method were not given and this makes it impossible to know the absolute accuracy of the theory. The author and his colleagues have obtained agreement better than $10 \%$ between computations using a method based on that described by Sinha et al. [2] and careful experimental measurements incorporating corrections for space harmonics, finite perturbing rod diameter and transverse field components.

Numerical Analysis: has been reported by Ward et al [3] and by Kory and Dayton [4]. All numerical analysis relies on discretisation of the structure to be analysed and very large numbers of elements must be used to obtain good accuracy. In these methods polar meshes have been used which are not an ideal fit to the boundaries of the helix or the support rods. The helix must, therefore, be represented by a staircase of elements. This could, presumably be avoided by using a finite element method with trapezoidal elements but that has not yet been reported. All numerical methods suffer from the difficulty of modelling the electric fields accurately close to the corners of the tape. This can lead to appreciable errors in the computation of the power flow in the structure and, hence, in the interaction impedance. Kory and Dayton reported agreement with experimental results to within $15 \%$ for a structure without dispersion shaping. The details of the experimental method were not given.

Experiment. The most accurate experimental methods are those described by Lagerstrom [5] which employ perturbation of the waves propagating on the helix using dielectric or metal rods placed along the axis. It is difficult to make accurate experimental measurements on many practical slow-wave structures because of their small sizes and careful attention must be paid 
to the alignment of the perturbing rods within the structures and to matching the r.f. terminations of the structure. Lagerstrom recognised that it is necessary to make corrections for the finite size of the perturbing rod and for space harmonic and transverse fields. The author and his colleagues have made careful experimental measurements on a wide range of helical slow-wave structures [6]. These have shown that errors of up to $50 \%$ can be introduced if these corrections are not made. The correction for the effect of transverse fields was negligible for a dielectric rod but could be as high as $20 \%$ for a metal rod. The repeatability of the experimental results was better than $4 \%$. The theory for the correction for the finite size of the rod assumed that the fields outside the rod were unchanged by it. The author has shown in unpublished work that this assumption is not valid for the perturbation of a cavity resonator by a dielectric rod and it is likely that the same is true in this case and may lead to errors of up to $10 \%$.

In order to be able to determine the interaction impedance of a helix slow-wave structure with confidence we require agreement between at least two, and preferably all three, of the methods described above. At present this has not been achieved to better than around $20 \%$ partly because the details of the experimental methods, and therefore their accuracies, have not been reported. An added complication is the effect of any uncertainty in the values of the relative permittivities of the support rods and of the perturbing rods used in experimental measurements. Recent work by the author has cast doubt on the accuracy of the use of cavity perturbation measurements to obtain this information. This paper will report comparisons between all three methods which will show the extent of the agreement between them when the details of all methods are known.

\section{References}

[1] S. D'Agostino, F. Emma and C. Paoloni, "Accurate analysis of helix slow-wave structures", IEEE Trans. Electron Devices, vol. ED-45, no.7, pp.1605-13, July 1998.

[2] A.K. Sinha, R. Verma, R.K. Gupta, L. Kumar, S.N. Joshi, P.K. Jain and B.N. Basu, "Simplified tape model of arbitrarily loaded helical slow-wave structures of a travelling wave tube", IEE Proc.-H, vol. 139, no. 4, pp. 347-50, August 1992.

[3] K.D. Ward and J. Wlodarczyk, "Transmission line modelling of helix slow-wave structures" in IEDM Tech. Dig., 1993 p.157.

[4] C.L. Kory and J.A. Dayton Jr., "Accurate cold-test model of helical TWT slow-wave circuits", IEEE Trans. Electron Devices, vol.45, no. 4, pp.966-971, April 1998.

[5] R.P. Lagerstrom, "Interaction-impedance measurements by perturbation of travelling waves", Technical Report No.7, Electronics Laboratories, Stanford University, February $11,1957$.

[6] Wang, P., Carter, R.G. and Basu, B.N., "An improved technique for measuring the Pierce impedance of helix slow-wave structures", Proc. European Microwave Conference, Cannes, pp. 998-1003, September 1994 